\title{
The Mortality Risk of Elevated Serum Transferrin Saturation and Consumption of Dietary Iron
}

\author{
Arch G. Mainous III, PbD \\ Brian Wells, $M D^{1}$ \\ Peter J. Carek, $M D, M S^{1}$ \\ James M. Gill, MD, $M P H^{2}$ \\ Mark E. Geesey, MS
}

'Department of Family Medicine, Medical University of South Carolina, Charleston, SC

${ }^{2}$ Department of Family \& Community Medicine, Christiana Care Health System, Wilmington, Del
Conflicts of interest: none reported

\section{CORRESPONDING AUTHOR}

Arch G. Mainous III, PhD

Department of Family Medicine

Medical University of South Carolina

PO Box 250192

295 Calhoun Street

Charleston, SC 29425

mainouag@musc.edu

\begin{abstract}
BACKGROUND Recent data shows an increased mortality risk associated with elevated transferrin saturation. Because ingestion of dietary iron may contribute to iron overload in persons with elevated transferrin saturation, we investigated the relationship between elevated transferrin saturation, ingestion of dietary iron and red meat, and mortality.
\end{abstract}

METHODS This 12-year cohort study used data from the second National Health and Nutrition Examination Survey 1976-1980 (NHANES II) and the NHANES II Mortality Study 1992. Population estimates were based on 9,229 persons aged 35 to 70 years at baseline. A Cox proportional hazards analysis was performed based on levels of transferrin saturation, intake of dietary iron, and intake of red meat. The analysis was conducted while controlling for demographics, severity of illness, body mass index, and smoking status.

RESULTS Unadjusted analyses indicated that those who had a high transferrin saturation and reported high dietary iron or red meat consumption had an increased mortality risk. The adjusted survival analysis indicated that persons with elevated transferrin saturation who reported high dietary iron intake had a hazard ratio for death of 2.90 (95\% confidence interval [Cl], 1.39-6.04) compared with those with normal transferrin saturation levels and reported low dietary iron intake. Persons who had a high transferrin saturation and reported high red meat consumption also had an increased hazard ratio for death $(2.26 ; 95 \% \mathrm{Cl}, 1.45-3.52)$ compared with those who had normal transferrin saturation and reported low red meat consumption.

CONCLUSIONS Ingestion of large quantities of dietary iron and red meat in persons with high transferrin saturation is associated with an increase in mortality. Simple dietary restrictions may reduce the mortality risk associated with high transferrin saturation.

Ann Fam Med 2004;2:139-144. DOI: 10.1370/afm.82.

\section{INTRODUCTION}

S erum transferrin saturation is a widely used marker for iron overload conditions such as hemochromatosis and African iron overload disease. ${ }^{1,2}$ The pathologic iron accumulation associated with these diseases affects many organs, including the liver, pancreas, and heart. ${ }^{1}$ Although few individuals have diagnosed iron overload conditions, such as hemochromatosis, the prevalence of elevated serum transferrin saturation ranges from $1 \%$ to $6 \%$ of the adult US population. ${ }^{3,4}$

Recent data suggest that elevated serum transferrin saturation carries an increased overall mortality risk. ${ }^{5}$ To prevent the complications of iron overload, some experts recommend that persons with elevated serum transferrin saturation moderate their consumption of red meats and organ meats. ${ }^{6}$ These dietary restrictions are thought to be unnecessary, however, for patients who have hemochromatosis and are undergoing therapeutic phlebotomies to control their iron levels. ${ }^{7}$ 
It is unknown whether the ingestion of a high iron diet increases the overall mortality risk among persons with elevated serum transferrin saturation. Accordingly, the purpose of this study is to examine the impact on mortality risk of a diet high in iron among persons with elevated serum transferrin saturation.

\section{METHODS}

\section{Data Sets}

We conducted an analysis of the cohort available in the second National Health and Nutrition Examination Survey 1976-1980 (NHANES II) and merged with the NHANES II Mortality Study, 1992. The NHANES II is a nationwide probability sample with adjusted sampling weights for 76 age-sex income groups designed to reflect closely the target population at the midpoint of the survey. The NHANES II Mortality Study (NH2MS) is a prospective cohort study that passively followed up on a subset of participants in the NHANES II. The NH2MS cohort is comprised of adults who were 30 to 75 years of age at the time of their NHANES II examination $(\mathrm{n}=9,252)$.

The NH2MS involves searching national databases containing information about mortality and causes of death. Mortality status was ascertained by computerized matching to national databases and evaluation of the resulting matches. Persons not found to be deceased were assumed alive for analytic purposes. The cohort study was terminated in December 1992. All living survey participants had been observed for at least 154 months, and our survival analysis was carried out to that point.

For the present study we excluded pregnant women, as well as the 2 respondents in the NHANES II who indicated that they had been told by a physician that they have hemochromatosis. These 2 respondents were excluded because they might have received treatment for their hemochromatosis. These exclusions resulted in a cohort of 9,229.

The data used in this study are public use data and are therefore exempt from requirement to receive an institutional review board approval.

\section{Variables}

\section{Transferrin Saturation}

In the NHANES II, the serum transferrin saturation was calculated by the personnel at the National Center for Health Statistics by dividing the serum iron level by total iron-binding capacity. Both serum ferritin and transferrin saturation are used to measure body iron stores. The serum ferritin level varies more with time than transferrin saturation and is therefore not as useful a marker by itself as transferrin saturation. We defined elevated serum transferrin saturation as greater than $55 \%$. This level was discovered to be related to all-cause mortality and is suggestive of hemochromatosis. ${ }^{5,8}$

Only a limited sample of NHANES II cohort had measured ferritin levels. Of our cohort of 9,229 persons, only 2,627 had measured ferritin levels. An iron overload group, defined as having both elevated transferrin saturation and elevated ferritin levels, contained only 9 persons. The NHANES II guidelines include a caution about conducting statistical analyses using the ferritin results because of the small population size. We therefore decided not to include ferritin levels in our analyses.

\section{Iron Ingestion}

Dietary iron comes in the 2 major forms of heme and nonheme iron. The largest dietary source of heme iron is red meat, whereas dietary supplements and vegetables mainly contain iron in the nonheme form. Because heme iron is much more readily absorbed by the gastrointestinal tract than nonheme iron, iron ingestion was measured in 2 ways. First, using the 24-hour dietary recall found in the NHANES II, the total iron intake (milligrams per day) was estimated by the National Center for Health Statistics. The United States recommended daily allowance for iron is $8 \mathrm{mg}$ for postmenopausal women, $8 \mathrm{mg}$ for all men, and $18 \mathrm{mg}$ for premenopausal women. ${ }^{10}$ Previous studies calculated a potential risk from excessive iron intake of 25 to $50 \mathrm{mg} / \mathrm{d} .{ }^{10,11}$ For the present study, more than $18 \mathrm{mg}$ of iron per day was considered high intake.

Second, to provide a general estimate of heme iron intake rather than total iron, each participant was asked the weekly frequency of eating various types of meat. Red meat consumption at least 7 times per week was considered high consumption.

\section{Mortality}

Mortality was measured as all-cause mortality. Mortality status was ascertained solely by computerized matching to national databases and evaluation of the resulting matches.

\section{Control Variables}

We examined the independent relationship of elevated serum transferrin saturation and dietary iron on mortality by controlling for potential confounders. Control variables that were available in the NHANES II baseline were age, race, sex, poverty status, and education. Three age categories were defined ( 30 to 50 years, 51 to 64 years, and older than 64 years). Race follows the NHANES II designations (white, black, other). Insurance status was not available in this data set, but the poverty income ratio (PIR), which is an index based on income and number of household members, was available. Poverty status was defined as below or above the poverty level (PIR $=1.0$ ). Because of our focus on all-cause mortality, we also included body mass index (BMI) and current self-reported smoking sta- 
tus. BMI was calculated from measured height and weight information with values higher than 30 defining obesity.

In an effort to control for severity of illness, we included self-reported health status as well as comorbid conditions. A variety of conditions were assessed in the NHANES II. Comorbidities were positive responses in the baseline interview to questions regarding whether a physician ever told the patient that he or she had each of the following conditions: cirrhosis, diabetes, high blood pressure, heart failure, heart attack, stroke, hardening of the arteries, rheumatic fever, rheumatic heart disease, heart murmur, ulcer (peptic, stomach, duodenal), chronic enteritis, ulcerative colitis, spastic colon or mucous colitis, gallstones, hepatitis, yellow jaundice, pleurisy, low blood pressure, cataracts, glaucoma, thyroid disease, polio or paralysis, goiter, hiatus hernia of the diaphragm, cancer, benign tumor, trouble with blood not clotting properly, loss of blood from stomach or bowels, nervous breakdown, neck injury, back injury, anemia, arthritis, gout, asthma, chronic bronchitis, emphysema, tuberculosis, and kidney problems. The Charlson Comorbidity Index was calculated from the responses to these questions. This index is a validated tool for predicting mortality in longitudinal studies. ${ }^{12}$

\section{Data Analysis}

We classified the population into 4 groups based upon normal and elevated transferrin saturation, and low and high iron intake. The criteria for high iron intake (total iron and red meat consumption) were analyzed separately.

For the analysis of the mortality, we used sampling weights to calculate prevalence estimates for the civilian noninstitutionalized US population. Because of the complex sampling design of the survey, we performed all analyses using the statistical software package SUDAAN, as recommended by the National Center for Health Statistics. Using the population estimates generated by SUDAAN, we computed Kaplan-Meier curves to show graphically the unadjusted relationship between allcause mortality and elevated serum transferrin saturation and low or high iron ingestion. We performed Cox proportional hazards analysis with all-cause mortality for serum transferrin saturations of greater than $55 \%$ controlling for age, race, sex, poverty status, education, BMI, smoking status, and Charlson Comorbidity Index. In these models, survival time was a continuous variable measured in 1-month increments up to 154 months from the baseline.

\section{RESULTS}

The data indicate that $1.1 \%$ of the adult US population aged 30 to 75 years at the time of the NHANES II (1976-1980) had a serum transferrin saturation above $55 \%$. Chi-square statistics were used to assess the relationship between transferrin saturation and a variety of demographic variables. Age was significantly related to the likelihood of having elevated transferrin saturation, with a larger proportion of younger persons having elevated transferrin saturation (Table 1). Self-reported health status and the Charlson Comorbidity Index

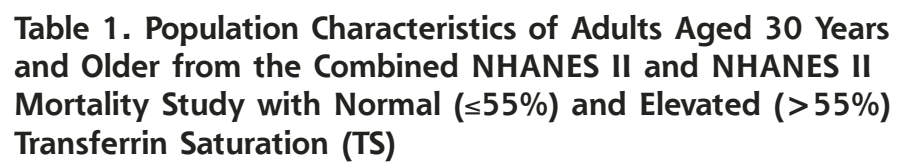

Table 1. Population Characteristics of Adults Aged 30 Years and Older from the Combined NHANES II and NHANES II Mortality Study with Normal $(\leq 55 \%)$ and Elevated ( $>55 \%$ ) Transferrin Saturation (TS)

\begin{tabular}{|c|c|c|c|}
\hline Characteristics & $\begin{array}{c}\text { Percent With } \\
\text { Normal TS } \\
\text { (n }=95,284,772)\end{array}$ & $\begin{array}{c}\text { Percent With } \\
\text { Elevated TS } \\
(n=1,073,696)\end{array}$ & $P$ Value \\
\hline \multicolumn{4}{|l|}{ Age group } \\
\hline $30-50 y$ & 54.3 & 69.1 & .02 \\
\hline $51-64$ y & 30.6 & 19.7 & \\
\hline$>64 y$ & 15.1 & 11.2 & \\
\hline \multicolumn{4}{|l|}{ Race } \\
\hline White & 88.0 & 86.6 & .88 \\
\hline Black & 9.6 & 9.5 & \\
\hline Other & 2.4 & 3.9 & \\
\hline \multicolumn{4}{|l|}{ Sex } \\
\hline Male & 47.5 & 48.4 & .87 \\
\hline Female & 52.5 & 51.6 & \\
\hline \multicolumn{4}{|c|}{ Poverty income ratio } \\
\hline$<1.0$ & 14.0 & 10.2 & .27 \\
\hline$\geq 1.0$ & 86.0 & 89.8 & \\
\hline \multicolumn{4}{|l|}{ Education level } \\
\hline Not hs graduate & 35.8 & 26.4 & .15 \\
\hline Hs graduate & 33.8 & 35.5 & \\
\hline Some college & 30.4 & 38.1 & \\
\hline \multicolumn{4}{|l|}{ Body mass index } \\
\hline$<30 \mathrm{~kg} / \mathrm{m} 2$ & 85.3 & 94.6 & $<.01$ \\
\hline$\geq 30 \mathrm{~kg} / \mathrm{m} 2$ & 14.7 & 5.4 & \\
\hline \multicolumn{4}{|l|}{ Current smoker } \\
\hline No & 60.1 & 40.6 & $<.01$ \\
\hline Yes & 39.9 & 59.4 & \\
\hline \multicolumn{4}{|l|}{ Health status } \\
\hline Excellent & 23.3 & 22.4 & .70 \\
\hline Very good & 24.7 & 28.6 & \\
\hline Good & 30.3 & 26.1 & \\
\hline Fair & 15.1 & 18.6 & \\
\hline Poor & 6.6 & 4.3 & \\
\hline \multicolumn{4}{|c|}{$\begin{array}{l}\text { Charlson comorbidity } \\
\text { index }\end{array}$} \\
\hline 0 & 16.1 & 22.7 & .54 \\
\hline 1 & 16.1 & 18.2 & \\
\hline 2 & 17.2 & 13.5 & \\
\hline$>2$ & 50.6 & 45.6 & \\
\hline
\end{tabular}


Figure 1. Kaplan-Meier survival curves for normal vs elevated transferrin saturation (TS) and low vs high iron intake.

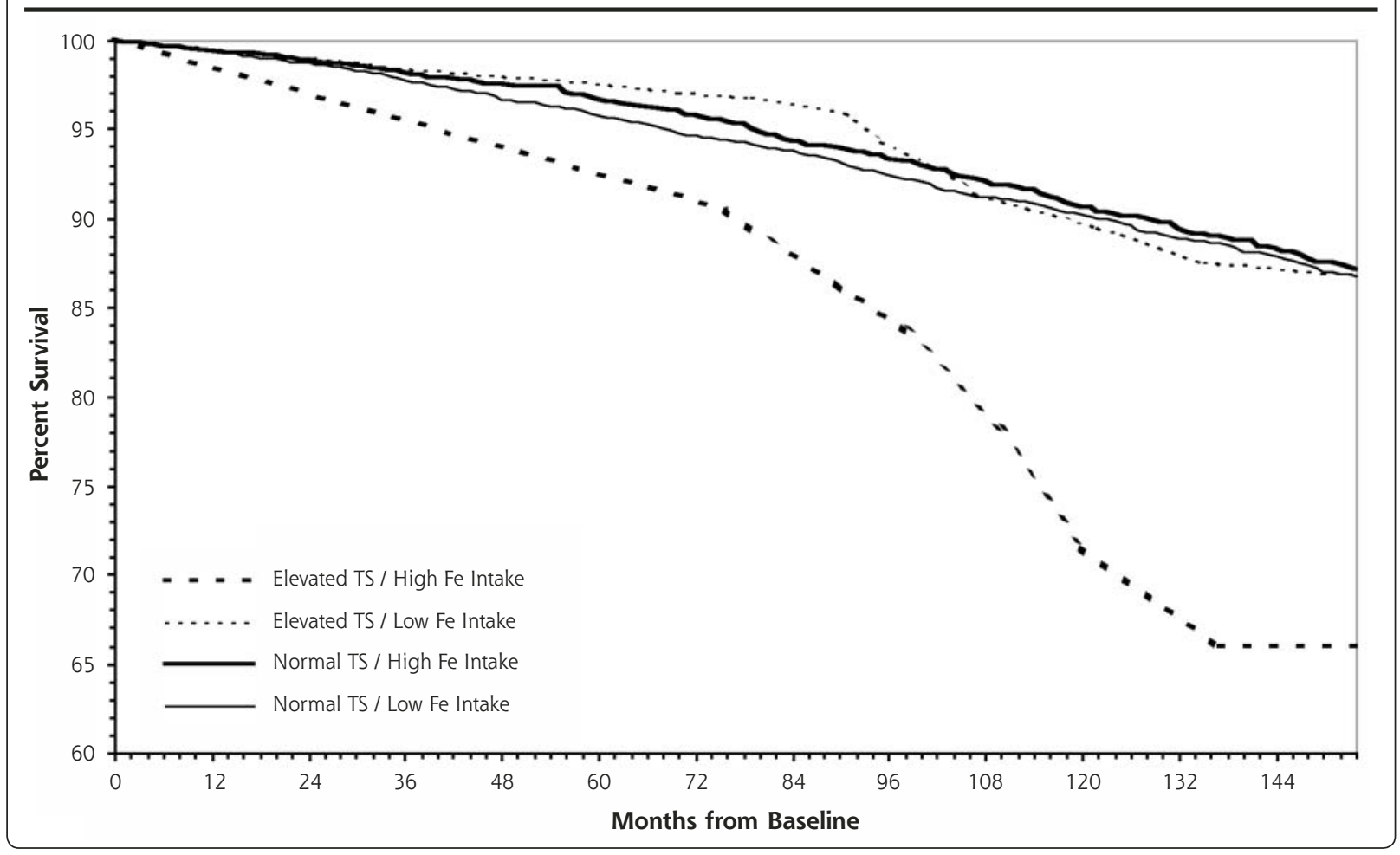

Figure 2. Kaplan-Meier survival curves for normal vs elevated transferrin saturation (TS) and low vs high red meat consumption.

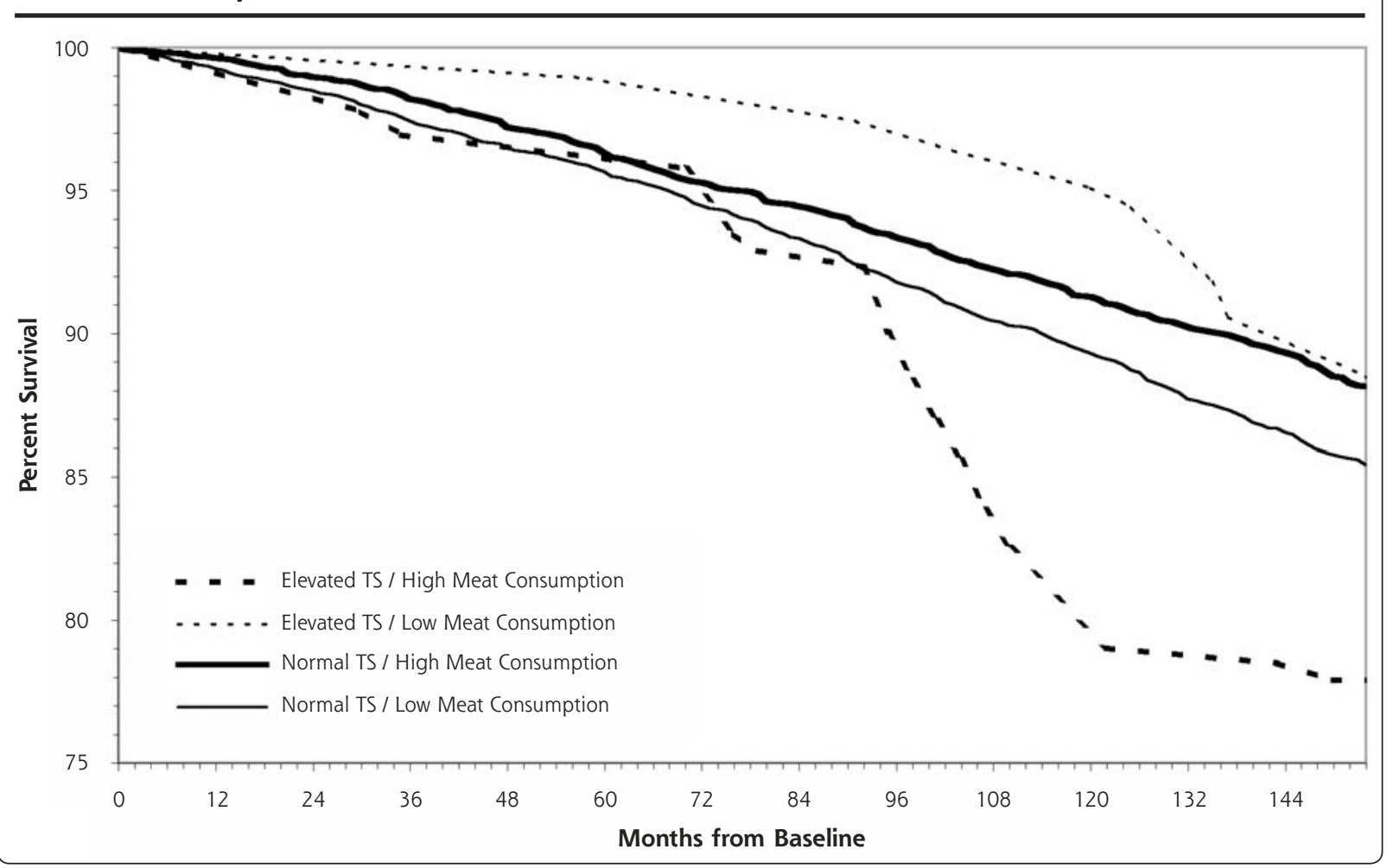


were not significantly related to elevated transferrin saturation.

Persons with elevated levels were less likely to consume high amounts of iron daily than were those with normal levels. For those with normal transferrin saturation, $81.8 \%$ had iron intake of $18 \mathrm{mg} / \mathrm{d}$ or less, whereas for those with elevated transferrin saturation, $88.4 \%$ had iron intake of $18 \mathrm{mg} / \mathrm{d}$ or less $(P=.05)$. No significant trends relating red meat consumption to elevated transferrin saturation levels at baseline were found. Among individuals with normal transferrin saturation, $46.8 \%$ consumed red meat fewer than 7 times per week, whereas $52.2 \%$ with elevated transferrin saturation consumed red meat fewer than 7 times per week $(P=.44)$

The unadjusted relationships between dietary iron intake and red meat consumption and survival among persons with elevated transferrin saturation are displayed in Figures 1 and 2. The results from the Cox proportional hazards model analysis of all-cause mortality by total dietary iron intake show that, when compared with persons with normal levels and low dietary iron intake, those with elevated levels and high dietary iron intake have a mortality risk 2.90 times greater (Table 2). Persons with elevated transferrin saturation but low intake of iron and those with normal levels but high iron intake were not significantly different from the group with normal transferrin saturation and low iron intake. Similarly, persons with elevated transferrin saturation who consume red meat 7 or more times per week were found to have an adjusted mortality risk 2.26 times greater than persons who have normal transferrin saturation and consume red meat fewer than 7 times per week.

\section{DISCUSSION}

The data from this 12-year cohort study indicate that persons with an elevated transferrin saturation and higher iron intake have a significantly increased allcause mortality risk. This finding was consistent both for total iron intake and for intake of heme iron ingested as a result of eating red meats. These increased mortality risks remained even after adjusting for important confounding variables. Additionally, this study provides some evidence regarding the mortality risk from the intake of red meat, which appears to be affected by whether the person has an elevated transferrin saturation.

High iron intake led to an increased mortality risk only when it was combined with elevated transferrin saturation. Persons with elevated transferrin saturation and normal iron intake did not have higher mortality risk. Similarly, those with normal transferrin saturation and either high iron or red meat intake did not have a higher mortality risk than those with normal transferrin saturation and normal iron intake.

Iron overload can lead to a variety of medical problems. Hemochromatosis is initially defined clinically by elevated transferrin saturation and represents a propensity to develop iron overload. It makes sense, therefore, that persons with a propensity for iron overload would have higher mortality rates only if they ingest greater amounts of iron. A diet high in iron would appear to be necessary but not sufficient to cause an increased mortality risk. These findings suggest, therefore, that persons with elevated transferrin might minimize their risk of disease complications simply by consuming a diet low in iron. Although the current recommendation for treatment of diagnosed iron overload syndromes is regular phlebotomy, the ability to use either a measure of transferrin saturation or genetic testing (ie, HFE gene with the $\mathrm{C} 282 \mathrm{Y}$ mutation) to stratify risk of iron overload might allow for selected individuals to be treated with dietary restrictions only.

This study has several strengths and limitations. The first strength is the longitudinal nature of the design. The mortality risk from iron ingestion was assessed by observing the cohort for more than 12 years, although greater effects might have been more evident had the observation period been extended. Second, by using the NHANES II data, we were able to generalize beyond a local sample of individuals to make national population estimates for the United States. Third, the cohort being studied was limited to persons 30 to 75 years of age at baseline. This agegroup allowed us to dovetail with what some have characterized as the classical age of onset of hemochromatosis at 40 to 60 years; hemochromatosis is the most common iron overload disease in the United States. ${ }^{13}$ 
Limitations of the study include use of a 24-hour dietary history to assess iron intake. Assessment of the exposure for total dietary iron was made only once and by recall rather than objective measurement. We have no information on dietary patterns after the baseline, and the diet may not have remained stable. Other studies, however, have shown that middle-aged people are likely to have a stable nutrient intake for many years. ${ }^{14,15}$ Second, although we measured elevated transferrin saturation, we were unable to assess iron overload with serum ferritin because of data collection issues in the NHANES II. We were also able to assess transferrin saturation only once. Transferrin saturation, however, is considered to be more stable with time than serum levels of ferritin. Third, we focused on allcause mortality rather than mortality from causes that would be predicted as consequences of iron overload. It has been contended that all-cause mortality is a more unbiased endpoint than disease-specific mortality. ${ }^{16}$ Additionally, it is possible that some individuals died during the course of the study but were not identified as deceased in the computerized databases. As a result, there is a potential for misclassification of individual status. Even so, there are no obvious reasons to suspect an unbalanced misclassification. Finally, calculations of the Charlson Comorbidity Index scores were based on patient recall, which might not accurately reflect the participants' true disease states.

In conclusion, the findings of this study have important clinical implications. First, this study suggests that high iron intake can cause problems even for those with elevated transferrin saturation who might have no clinical complications and who might not have had hemochromatosis diagnosed. As clearly shown, persons with an elevated transferrin saturation who ingest normal levels of iron or red meat have the same mortality risk as those with a normal transferrin saturation. Thus, an easy treatment program might eventually involve focusing on dietary restrictions instead of or in addition to therapeutic phlebotomy.

Second, this study provides some evidence regarding the mortality risk from the intake of red meat. One study showed in a 12-year cohort, using a model adjusting for BMI, smoking, and socioeconomic status, that in comparison with those who eat meat, those who do not eat red meat had a decreased mortality risk. ${ }^{17}$ The aforementioned study did not account for iron status, however. Our results suggest that the risk of high red meat intake is limited to those with a predisposition to iron overload, eg, individuals with high transferrin saturation. Future research is necessary to determine more fully the relationship among elevated transferrin saturation, iron overload, and potential factors that might affect mortality.
To read commentaries or to post a response to this article, see it online at http://www.annfammed.org/cgi/content/full/2/2/139.

Key words: Transferrin; mortality; cohort analysis

Submitted April 1, 2003; submitted, revised, July 2, 2003; accepted August 6, 2003.

Funding support: This study was funded in part through grant 1D12HP00023-01 from the Health Resources and Services Administration.

\section{References}

1. McCullen MA, Crawford DHG, Hickman PE. Screening for hemochromatosis. Clinica Chimica Acta. 2002;315:169-186.

2. Barton JC, Edwards CQ, Bertoli LF, Shroyer TW, Hudson SL. Iron overload in African Americans. Am J Med. 1995;99:616-623.

3. Looker AC, Johnson CL. Prevalence of elevated serum transferrin saturation in adults in the United States. Ann Intern Med. 1998;129: 940-945.

4. Mainous AG III, Gill JM, Pearson W/S. Should we screen for hemochromatosis? An examination of evidence of downstream effects on morbidity and mortality. Arch Intern Med. 2002;162:1769-1774.

5. Mainous AG III, Gill JM, Carek, PJ. Elevated serum transferrin saturation and mortality. Ann Fam Med. 2004;2:133-138.

6. Barton JC, McDonnell SM, Adams PC, et al. Management of hemochromatosis. Ann Intern Med. 1998;129:932-939.

7. Witte DL, Crosby WH, Edwards CQ, Fairbanks VF, Mitros FA. Practice guideline development task force of the College of American Pathologists. Hereditary hemochromatosis. Clin Chim Acta. 1996;245: 139-200.

8. McLaren CE, McLachlan G, Halliday J, et al. Distribution of transferrin saturation in an Australian population: relevance to the early diagnosis of hemochromatosis. Gastroenterology. 1998;114:543-549.

9. McCarthy GM, McCarthy CJ, Kenny D, Crowe J, Eustace S. Hereditary hemochromatosis: a common, often unrecognized, genetic disease. Clev Clin J Med. 2002;69:224-237.

10. Standing Committee on the Scientific Evaluation of Dietary Reference Intakes, Food and Nutrition Board, Institute of Medicine. Dietary Reference Intakes for Vitamin A, Vitamin K, Arsenic, Boron, Chromium, Copper, Iodine, Iron, Manganese, Molybdenem, Nickel, Silicon, Vanadium, and Zinc: a Report on the Panel of Micronutrients. Washington, DC: National Academy Press; 2001:290-393.

11. Schumann K. Safety aspects of iron in food. Ann Nutrit Metab. 2001;45:91-101.

12. Charlson ME, Pompei P, Ales KL, MacKenzie CR. A new method of classifying prognostic comorbidity in longitudinal studies: development and validation. J Chronic Dis. 1987;40:373-83.

13. Niederau C, Fischer R, Purshel A, Stremmel W, Haussinger D, Strohmeyer $\mathrm{G}$. Long-term survival in patients with hemochromatosis. Gastroenterology. 1996;110:1107-1109.

14. Jensen OM, Whrendorf J, Rosenquist A, Geser A. The reliability of questionnaire-derived historical dietary information and temporal stability of food habits in individuals. Am J Epidemiol. 1984;120:281290.

15. James GD, Sealey JE, Alderman MH, Laragh JH. Year to year stability of urine sodium, potassium, aldosterone and PRA in normotensive men and women. Am J Hypertens. 1993;6:86A-90A.

16. Black WC, Haggstrom DA, Welch HG. All-cause mortality in randomized trials of cancer screening. J Natl Cancer Inst. 2002;94:167-173.

17. Appleby PN, Throgood M, Mann JI, Key TJ. The Oxford Vegetarian Study: an overview. Am J Clin Nutr. 1999;70:525S-531S. 